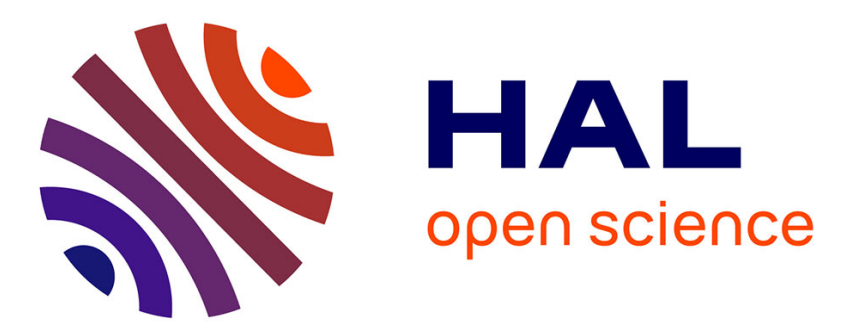

\title{
Column generation algorithms for exact modularity maximization in networks
}

\author{
Daniel Aloise, Sonia Cafieri, Gilles Caporossi, Pierre Hansen, Leo Liberti, \\ Sylvain Perron
}

\section{- To cite this version:}

Daniel Aloise, Sonia Cafieri, Gilles Caporossi, Pierre Hansen, Leo Liberti, et al.. Column generation algorithms for exact modularity maximization in networks. Physical Review E : Statistical, Nonlinear, and Soft Matter Physics, 2010, 82 (4), pp 046112-1 - 046112-9. 10.1103/PhysRevE.82.046112 . hal00934661

\section{HAL Id: hal-00934661 \\ https://hal-enac.archives-ouvertes.fr/hal-00934661}

Submitted on 15 Apr 2014

HAL is a multi-disciplinary open access archive for the deposit and dissemination of scientific research documents, whether they are published or not. The documents may come from teaching and research institutions in France or abroad, or from public or private research centers.
L'archive ouverte pluridisciplinaire HAL, est destinée au dépôt et à la diffusion de documents scientifiques de niveau recherche, publiés ou non, émanant des établissements d'enseignement et de recherche français ou étrangers, des laboratoires publics ou privés. 
Column Generation Algorithms for Exact Modularity Maximization in Networks

D. Aloise, S. Cafieri,

G. Caporossi, P. Hansen,

L. Liberti, S. Perron

G-2010-36

June 2010

Les textes publiés dans la série des rapports de recherche HEC n'engagent que la responsabilité de leurs auteurs. La publication de ces rapports de recherche bénéficie d'une subvention du Fonds québécois de la recherche sur la nature et les technologies. 



\title{
Column Generation Algorithms for Exact Modularity Maximization in Networks
}

\author{
Daniel Aloise ${ }^{a}$ \\ daniel.aloise@gerad.ca \\ Sonia Cafieri ${ }^{b}$ \\ sonia.cafieri@enac.fr \\ Gilles Caporossi ${ }^{c}$ \\ gilles.caporossi@gerad.ca \\ Pierre Hansen ${ }^{c, d}$ \\ pierre.hansen@gerad.ca \\ Leo Liberti ${ }^{d}$ \\ liberti@lix.polytechnique.fr \\ Sylvain Perron ${ }^{c}$ \\ sylvain.perron@gerad.ca \\ ${ }^{a}$ Dept. of Production Engineering \\ Universidade Federal do Rio Grande do Norte \\ Campus Universitrio s/n, Natal-RN, Brazil, 59072-970 \\ ${ }^{b}$ Département de Mathmatiques et Informatique \\ École Nationale de l'Aviation Civile \\ 7 ave. E. Belin, F-31055 Toulouse, France \\ ${ }^{c}$ GERAD \& HEC Montréal \\ 3000, chemin de la Côte-Sainte-Catherine \\ Montréal (Québec) Canada, H3T 2AY \\ d LIX, École Polytechnique \\ F-91128 Palaiseau, France
}

June 2010

Les Cahiers du GERAD

G-2010-36

Copyright (c) 2010 GERAD 



\begin{abstract}
Finding modules, or clusters, in networks currently attracts much attention in several domains. The most studied criterion for doing so, due to Newman and Girvan [Physical Review E 69, 026113 (2004)], is modularity maximization. Many heuristics have been proposed for maximizing modularity and yield rapidly near optimal solution or sometimes optimal ones but without a guarantee of optimality. There are few exact algorithms, prominent among which is a paper by $\mathrm{Xu}$ et al. [Eur. Physical Journal B 60, 231-239 (2007)]. Modularity maximization can also be expressed as a clique partitioning problem and the row generation algorithm of Grötschel and Wakabayashi [Mathematical Programming 45, 59-96 (1989)] applied. We propose to extend both of these algorithms using the powerful column generation methods for linear and non linear integer programming. Performance of the four resulting algorithms are compared on problems from the literature. Instances with up to 512 entities are solved exactly for the first time. Moreover, the computing time of previously solved problems are reduced substantially.
\end{abstract}

\title{
Résumé
}

L'identification de modules, ou groupes, dans les réseaux attire actuellement beaucoup d'attention dans plusieurs domaines. Pour arriver à identifier les modules, le critère le plus utilisé jusqu'à présent est celui de la maximisation de la modularité, critère introduit par Newman et Girvan [Physical Review E 69, 026113 (2004)]. Plusieurs méthodes heuristiques ont été proposées pour ce critère et procurent rapidement une solution quasi optimale ou parfois une solution optimale sans toutefois une preuve de son optimalité. Parmi le très faible nombre de méthodes exactes proposées, la plus importante est très certainement celle proposée par Xu et al. [Eur. Physical Journal B 60, 231-239 (2007)]. La maximisation de la modularité peut également être exprimée comme un problème de partionnement en cliques pouvant être résolu par l'algorithme de génération de contraintes de Grötschel et Wakabayashi [Mathematical Programming 45, 59-96 (1989)]. Nous proposons d'étendre ces deux algorithmes en utilisant les techniques avancées de la génération de colonnes pour la programmation linéaire et non linéaire entière. La performance des quatre algorithmes est comparée sur des problèmes provenant de la littérature. Des instances contenant jusqu'à 512 entités sont résolues exactement pour la première fois. De plus, les temps de résolution pour des problèmes déjà résolus sont réduits substantiellement.

Acknowledgments: Financial support by Grants ANR 07-JCJC-0151 "ARS", Digiteo 2009-14D "RMNCCO", Digiteo 2009-55D "ARM", are greatefully acknowledged. P.H. acknowledges support from Digiteo Foundation. S.P. has been supported by the NSERC (Natural Sciences and Engineering Research Council of Canada) grant 327435-06. G.C., P.H. and S.P. would like to thank the research office of HEC Montréal for the participation to the 2009-2010 research workshop of HEC Montréal. 



\section{Introduction}

Clustering is an important chapter of data analysis and data mining with numerous applications in a variety of fields. It aims at solving the following general problem: given a set of entities, find subsets, or clusters, which are homogeneous and/or well-separated. As the concepts of homogeneity and of separation can be made precise in many ways, there are a large variety of clustering problems [1-4]. These problems in turn are solved by exact algorithms or, more often and particularly for large data sets, by heuristics. An exact algorithm provides, hopefully in reasonable computing time, an optimal solution together with a proof of its optimality. A heuristic provides, usually in moderate computing time, a near optimal solution or sometimes an optimal solution but without proof of its optimality.

In the last decade, clustering on networks has been extensively studied, mostly in the physics and computer science research communities. Rather than using the term cluster, the words module or community are often adopted in the physics literature. Recall that a network, or graph, $G=(V, E)$ is composed of a set $V$ of $n$ vertices and a set $E$ of $m$ edges, which join pairs of vertices. A vertex $v_{j}$ is represented by a point and an edge $e_{i j}=\left\{v_{i}, v_{j}\right\}$ by a line joining its two end vertices $v_{i}$ and $v_{j}$. The shape of this line does not matter, only the presence or absence of an edge is important. In a simple graph, there is at most one edge between any pair of vertices, otherwise one has a multigraph. With a slight abuse of set notation, a loop $e_{i i}=\left\{v_{i}, v_{i}\right\}$ is an edge for which both end vertices coincide. The degree $k_{i}$ of a vertex $v_{i} \in V$ is the number of edges incident with $v_{i}$. A subgraph $G_{S}=\left(S, E_{S}\right)$ of a graph $G=(V, E)$ induced by a set of vertices $S \subseteq V$ is a graph with vertex set $S$ and edge set $E_{S}$ equal to all edges with both vertices in $S$. Such a subgraph corresponds to a cluster (or module or community) and many heuristics aim at finding a partition of $V$ into pairwise disjoint nonempty subsets $V_{1}, V_{2}, \ldots, V_{N}$ inducing subgraphs of $G$. Various objective functions have been proposed for evaluating such a partition. Roughly speaking, one seeks modules which contain more inner edges (with both vertices in the same module) than cut edges (with vertices in different modules). The degree $k_{i}$ of the vertex $v_{i}$ can be split in two: the indegree $k_{i}^{i n}$ or number of neighbors within its community and the outdegree $k_{i}^{\text {out }}$ or number of neighbors outside its community. Several concepts of community follow.

In 2004, Radicchi et al. [5] defined a community in the strong sense as a subgraph all vertices of which have larger indegree than outdegree and a community in the weak sense as a subgraph for which the sum of vertex indegrees is larger than the sum of vertex outdegrees. As an inner edge contributes by two to the sum of the indegrees and a cut edge contributes by one to the sum of outdegrees, the number of inner edges in a community in the weak sense must be at least as large as half the number of cut edges. As cut edges contribute to the sum of degrees of two communities, this definition entails that for the network as a whole the number of inner edges is larger than the number of cut edges. Recently, several extensions of the definition of the community in the weak sense have been proposed. One may consider the difference for each community of the sum of indegrees and the sum of outdegrees. Then summing these contributions for all communities gives a multiway cut problem. Another approach is to normalize the contribution of each community by dividing it by its number of vertices [6]. The resulting function, to be maximized, is called modularity density. Alternatively, contributions of communities may be divided by their number of edges [7]. Finally, one may consider maximizing, in a divisive hierarchical method, the minimum ratio of the number of edges in a community divided by the number of cut edges [8].

A different, and currently mainstream, approach was inaugurated by Newman and Girvan also in 2004 [9]. They propose to find a partition of $V$ which maximizes the sum, over all modules, of the number of inner edges minus the expected number of such edges assuming that they are drawn at random with the same distribution of degrees as in $G$. In [9] the following precise definition of modularity is given:

$$
Q=\sum_{s}\left[a_{s}-e_{s}\right]
$$

where $a_{s}$ is the fraction of all edges that lie within module $s$ and $e_{s}$ is the expected value of the same quantity in a graph in which the vertices have the same expected degrees but edges are placed at random. A maximum value of $Q$ near to 0 indicates that the network considered is close to a random one (barring fluctuations), while a maximum value of $Q$ near to 1 indicates strong community structure. Observe that maximizing modularity gives an optimal partition together with the optimal number of modules. 
The modularity maximization problem has been extensively studied both from the algorithmic and from the applications viewpoints. Some papers discuss a few of its mathematical properties, among which are the following.

(i) Even if the networks under study most of the time have no loops nor multiple edges, the expected number of loops may be positive. Moreover, for some pairs of end vertices, their expected number of edges may be greater than 1 [10].

(ii) Modularity maximization suffers from a resolution limit [11], i.e., when the network is large small modules can be absorbed by larger ones even if they are very dense.

(iii) The partition with optimal or near optimal modularity may contain modules which are not communities in the weak sense, or even in the most weak sense [12] that twice the number of inner edges is never less than the number of edges joining this module to another one.

Modification to the model and/or to heuristics have been proposed to address these problems. Changing slightly the null model (i.e. the value of $e_{s}$ in Eq. (1)), by simulation [10] or by using an analytical formula [13] allows the removal of loops and multiple edges. A parameter can also be introduced together with multiresolution heuristics $[14,15]$. Nevertheless, the original modularity maximization appears presently to be much used. In this paper, we limit ourselves to this classical problem.

Brandes et al. [16] have shown that modularity maximization is NP-hard.

Numerous heuristics have been proposed to maximize modularity. They are based on divisive hierarchical clustering, agglomerative hierarchical clustering, partitioning, and hybrids. They rely upon various criteria for agglomeration or division [17-21], simulated annealing [10,22,23], mean field annealing [24], genetic search [25], extremal optimization [26], spectral clustering [27-29], linear programming followed by randomized rounding [30], dynamical clustering [31], multilevel partitioning [32], contraction-dilation [33], multistep greedy search [34], quantum mechanics [35] and other approaches [14, 21, 29,36-38].

In contrast, papers proposing exact algorithms or using mathematical programming are rare for modularity maximization. There are two approaches. In the first one, the original graph $G=(V, E)$ is replaced by a complete weighted graph $K_{n}=\left(V, E^{\prime}\right)$ of order $n=|V|$ as $G$ and such that for any pair of vertices $v_{i}, v_{j} \in G$, $K_{n}$ has an edge $e_{i j}^{\prime}$ with a weight equal to the modularity of that edge. In addition to the complexity result mentioned above, Brandes et al. [16] give an integer programming formulation for modularity maximization and mention that the optimal solution of two test problems with 34 and with 105 entities were determined. Their approach is in fact close to the work of Grötschel and Wakabayashi $[39,40]$ on clique partitioning. It is discussed in the next section. The second approach works directly on the original graph $G=(V, E)$. $\mathrm{Xu}$, Tsoka and Papageorgiou [41] propose a mixed integer convex quadratic programming model, discussed below. They solve exactly four test problems with up to 104 entities.

The purpose of the present paper is to assess and advance the state of the art of algorithms for exact modularity maximization. To this effect, we discuss and compare four exact algorithms, two of which are new. Two of these algorithms work on a reduction of modularity maximization to clique partitioning; the other two work on the direct formulation. They are: (i) the row generation algorithm of [39], which subsumes the algorithm of [16], (ii) a new stabilized column generation algorithm for clique partitioning which enhances the efficiency of that approach, (iii) the mixed integer convex quadratic programming approach of [41], (iv) another new stabilized column generation algorithm which enhances the efficiency of the second approach.

Column generation algorithms implicitly take into account all possible communities (or in other words all subsets of the set of entities under study). They replace the problem of finding simultaneously all communities in an optimal partition by a sequence of optimization problems for finding one community at a time, or more precisely a community which improves the modularity of the current solution. So, problems are solved much faster than with previous algorithms and larger instances can be tackled, the largest to date having 512 entities.

Clearly, in many applications of modularity maximization, instances are still larger and sometimes very much larger, than those which can be solved exactly with the proposed algorithms. Nevertheless, it is our belief that exact algorithms are worthy of study for several reasons. 
(i) Instances which are more than toy problems can presently be solved exactly. As shown below, these include many problems used to illustrate the performance of various heuristics.

(ii) As mentioned in several papers, finding the significance of the detected communities is difficult. Indeed, various heuristics often lead to partitions that disagree on two or more of their communities. Having an exact solution solves the problem of separating possible inadequacies of the model from eventual errors resulting from the use of heuristics. Unsuspected communities may be interpreted with more confidence and proposed to the user for a substantive analysis. This is one of the main aims of clustering.

(iii) If solving a given instance proves to be too time consuming, the exact algorithm may often be stopped and the best solution found considered as a heuristic one. It is not uncommon that the optimal solution is found at an early stage of the resolution. Then, the problem of maximizing modularity will, in fact, be solved but without a proof of optimality.

(iv) An exact algorithm can provide a benchmark of exactly solved instances which can be used to compare heuristics and fine tune them. More precisely, the comparison of the symmetric differences between the optimal solution and the heuristically obtained ones may suggest additional moves which improve the heuristic under study. Iterating this approach with several heuristics may lead to performant hybrids. As a rule of thumb, a sophisticated heuristic should be able to find quickly an optimal solution for most or possibly all practical instances which can be solved exactly with a proof of optimality.

(v) Using projection, i.e., fixing some of the communities found by a heuristic, an exact algorithm can be applied to this reduced network in order to improve the heuristic solution [42].

(vi) Getting improved heuristics will in turn lead to more efficient exact algorithms. Indeed, there are usually some steps in such algorithms which can be solved heuristically, always or most of the time, without forfeiting the guarantee of optimality. In column generation algorithms, as discussed below, knowledge of a good or possibly optimal initial solution enhances stabilization substantially. Heuristics are also useful in solving the auxiliary problem.

(vii) An exact algorithm can also be used as a tool for the theoretical study of maximum modularity partitions, e.g., the fact that they satisfy or not conditions on the number of inner and of cut edges of communities, or conditions of robustness [12].

(viii) With active research, regularly improved mathematical programming packages such as CPLEX [43] and increasing computer power, the size of problems solved exactly is likely to increase substantially with time. A comparison with a central problem of Operations Research, i.e., the traveling salesman problem, illustrates this point. Given a set of cities and pairwise distances between them, the traveling salesman problem is to find a minimum length tour visiting once and only once each city. In 1954, Dantzig, Fulkerson, and Johnson [44] solved optimally an instance with 49 cities. In 2009, Applegate et al. [45] were able to solve to optimality an instance with 85900 cities.

\section{Modularity maximization as clique partitioning}

\subsection{Row generation}

The modularity function was expressed above as a sum of values over all communities. As shown in [27], modularity can also be written as a sum of values over all edges of the complete graph $K_{n}$.

$$
Q=\frac{1}{2 m} \sum_{i, j \in V}\left(a_{i j}-\frac{k_{i} k_{j}}{2 m}\right) \delta\left(c_{i}, c_{j}\right)
$$

where $m$ corresponds to the cardinality of $E, k_{i}, k_{j}$ are the degrees of vertices $i$ and $j$, respectively, $a_{i j}$ is the $i j$ component of the adjacency matrix of $G$, equal to 1 if vertices $i$ and $j$ are adjacent, and to 0 otherwise. Finally, $\delta\left(c_{i}, c_{j}\right)$ is the Kronecker symbol equal to 1 if the communities $c_{i}$ and $c_{j}$, to which $i$ and $j$ belong, are the same, and to 0 otherwise. The quantity $\frac{k_{i} k_{j}}{2 m}$ is the expected number of edges between vertices $i$ and $j$ in a null model where edges are placed at random, while the distribution of degrees remains the same. 
Introducing binary variables $x_{i j}$ equal to 1 if vertices $i$ and $j$ belong to the same module and 0 otherwise, and setting

$$
w_{i j}=\frac{1}{m}\left(a_{i j}-\frac{k_{i} k_{j}}{2 m}\right),
$$

modularity maximization can be reformulated as a clique partitioning problem. Since $K_{n}$ is complete, it is a clique and any of its induced subgraphs are cliques also. Partitioning $G$ is thus equivalent to partitioning $K_{n}$ into cliques. The resulting partition is an equivalence relation, i.e., reflexive, symmetric and transitive. From reflexivity (or the fact that each entity is in the same module as itself) all $x_{i i}=1$. Thus the sum of elements on the main diagonal is a constant for all partitions, equal to

$$
-C=-\sum_{i \in V} \frac{k_{i} k_{i}}{2 m}
$$

From symmetry, using $x_{i j}=x_{j i}$, one can eliminate variables corresponding to values of indices $i>j$. The model can then be written, as in [39,40],

$$
\begin{array}{ll}
\max & \sum_{i<j \in V} w_{i j} x_{i j}-C \\
\text { s.t. } & x_{i j}+x_{j k}-x_{i k} \leq 1 \\
& x_{i j}-x_{j k}+x_{i k} \leq 1 \\
& -x_{i j}+x_{j k}+x_{i k} \leq 1 \\
& x_{i j} \in\{0,1\}
\end{array}
$$

for all $1 \leq i<j<k \leq n$, for all $1 \leq i<j<k \leq n$, for all $1 \leq i<j<k \leq n$, for all $1 \leq i<j \leq n$.

This model has $\frac{n(n-1)}{2}$ variables and $3\left(\begin{array}{l}n \\ 3\end{array}\right)=\frac{n(n-1)(n-2)}{2}=O\left(n^{3}\right)$ constraints. The first three sets of constraints express transitivity, i.e., if entities $i$ and $j$ are in the same module and entities $j$ and $k$ are in the same module, then entities $i$ and $k$ must be in the same module. The fourth (and last) set of constraints expresses integrality, i.e., edges are present in the solution entirely or not at all.

Problem (5) is a linear program in 0-1 variables and thus small instances may be solved by integer linear programming packages such as CPLEX. In the solution process, the linear programming relaxation obtained by replacing the constraints $x_{i j} \in\{0,1\}$ by $x_{i j} \in[0,1]$ is first solved. If the optimal solution of this relaxation is in integers, which is often the case, it corresponds to a partition of maximum modularity.

Should the solution of the continuous relaxation be fractional, one can branch, or add one or several cutting planes, i.e., additional linear constraints which cut off the current fractional solution but do not eliminate any feasible integer solution. Branching is done by setting a fractional $0-1$ variable $x_{i j}$ to 1 or 0 , i.e., imposing on the one hand that entities $i$ and $j$ belong to the same community and on the other hand that they belong to different communities. Two linear programming subproblems are thus obtained and their solution gives bounds valid for the former and the latter case respectively. These bounds are not larger than the bound given by the solution of the subproblem on which branching took place. Branching on a subproblem stops if the value of the corresponding bound is smaller than that of the best solution known, or incumbent. As the possible number of branching choices is finite, the algorithm converges. Choosing a variable $x_{i j}$ with value closest to $\frac{1}{2}$ balances improvement of both bounds. These rules and others are discussed in [46]. In practice, branching is done by CPLEX. Several families of cutting planes were obtained in $[39,40]$ and could be used as an alternative or complement to branching.

Note that a similar formulation is presented in [16] but with constraints of the type $x_{i j}+x_{j k}-2 x_{i k} \leq 1$ instead of $x_{i j}+x_{j k}-x_{i k} \leq 1$, and so forth. While this does not change the set of feasible solutions in $0-1$ variables, the continuous relaxation in the algorithm of [16] will be less tight than that of (5). Consequently, the algorithm is more time consuming than that of [39] as it is shown in our computational experiments (see Section 4 below).

Unfortunately, the number of constraints of problem (5) grows rapidly with $n$. Not all of these constraints will be tight at the optimum. Those which are not could be deleted without changing the optimal solution 
but it is not known a priori which ones they are. Grötschel and Wakabayashi [39] therefore proposed to add the constraints progressively. More precisely, they add by batches of 300 those constraints which are most violated by the current solution. In their experiments on a variety of clustering problems, they found that only a small number of such constraints were tight at the optimum and that the solution of the continuous relaxation was very often integer. Our computation experiments (see Section 4 below) show this appears also to be the case for modularity maximization.

\subsection{Column generation}

Column generation is a powerful technique of linear programming which allows the exact solution of linear programs with a number of columns exponential in the size of the input (there may be billions of them and, in some cases, much more). To this effect, it follows the usual steps of the simplex algorithm, apart from finding an entering column with a positive reduced cost in case of maximization which is done by solving an auxiliary problem. The precise form of this last problem depends on the type of problem under study. It is often a combinatorial optimization or a global optimization problem. It can be solved heuristically as long as a column with a reduced cost of the required sign can be found. When this is no longer the case, an exact algorithm for the auxiliary problem must be applied either to find a column with the adequate reduced cost sign, undetected by the heuristic, or to prove that there is no such column and hence the linear programming relaxation is solved. Column generation has proven to be very useful in the solution of large clustering problems, e.g., minimum sum-of-squares clustering $[1,47,48]$.

For modularity maximization clustering, as for other clustering problems with an objective function additive over the clusters, the columns correspond to all subsets of $V$, i.e., to all nonempty modules.

To express this problem, define $a_{i t}=1$ if vertex $i$ belongs to module $t$ and to $a_{i t}=0$ otherwise. One can then write the model as

$$
\begin{aligned}
& \max \sum_{t \in T} c_{t} z_{t}-C \\
& \text { s.t. } \sum_{t \in T} a_{i t} z_{t}=1 \\
& \forall i=1, \ldots, n \\
& z_{t} \in\{0,1\} \\
& \forall t \in T,
\end{aligned}
$$

where $c_{t}=\sum_{i} \sum_{j>i} w_{i j} a_{i t} a_{j t}$, i.e., the value of the module indexed by $t$ with $t=1 \ldots 2^{n}-1$.

The objective function (6) of the primal problem expresses that modularity is equal to the sum of modularities of all selected modules minus a constant corresponding to the diagonal terms. The first set of constraints (7) expresses that each entity must belong to one and only one module and the second set of constraints that modules must be selected entirely or not at all.

If the integrality constraints (8) are replaced by

$$
z_{t} \geq 0, \forall t \in T
$$

the upper bound $z_{t} \leq 1$ being implied by constraint (7), one obtains a relaxation of (6)-(8) which is a linear program.

Recall that to any primal linear program is associated another linear program called its dual. This dual program has as many variables as the primal has constraints and as many constraints as the primal has variables.

The dual of the relaxation of $(6)-(7),(9)$ can be written

$$
\begin{array}{ll}
\min & \sum_{i=1}^{n} \lambda_{i}-C \\
\text { s.t. } & \sum_{i=1}^{n} a_{i t} \lambda_{i} \geq c_{t}
\end{array} \quad \forall t \in T,
$$




$$
\lambda_{i} \in \mathbb{R} \quad \forall i=1 \ldots n .
$$

The objective function (10) of the dual problem (10)-(12) is equal to the sum of all dual variables minus a constant $C$. The constraints (11) express that the sum of dual variables associated with the entities of any community must be at least as large as its modularity. Finally, the constraints (12) express the fact that the dual variables are unrestricted in sign.

From the duality theorem of linear programming, the optimal solutions $\left(z_{1}^{*}, z_{2}^{*}, \ldots, z_{T}^{*}\right)$ of the primal and $\left(\lambda_{1}^{*}, \lambda_{2}^{*}, \ldots, \lambda_{n}^{*},\right)$ of the dual have the same value:

$$
\sum_{t \in T} c_{t} z_{t}^{*}=\sum_{i=1}^{n} \lambda_{i}^{*}
$$

Moreover, any feasible solution of the primal has smaller or equal value than any solution of the dual. Geometrically, the sets of feasible solutions of the primal and dual are polyhedra. Finding the optimal solution of the primal or of the dual amounts to finding an optimal vertex and the corresponding cone in either of these polyhedra.

Problem (6)-(7),(9) is called the master problem. It is the relaxation of a partitioning problem which can in principle be solved by a package such as CPLEX. However, as the number of columns is exponential, this is possible only for very small $n$. To overcome these difficulties, one resorts to column generation. Following that approach a reduced master problem with considerably fewer columns is solved instead of the full master problem (6)-(7),(9). As usual in the branch and bound approach to mixed integer programming, one first solves the continuous relaxation of this restricted master problem. One begins with a relaxed problem containing some feasible columns and possibly artificial variables. Then improving columns will be added progressively. Finding such column(s) is the auxiliary problem whose role is to find a column with positive (negative) reduced cost in case of maximization (minimization). For problem (6)-(7),(9), the reduced cost associated with column $t$ will be equal to $c_{t}-\sum_{i} \lambda_{i} a_{i t}$ where the $\lambda_{i}$ are the current values of the dual variables of the continuous relaxation of problem $(6)-(7),(9)$. Replacing the coefficients $a_{i t}$ by binary variables $y_{i}$ leads to the following expression of the auxiliary problem:

$$
\max _{y \in \mathbb{B}^{n}} \sum_{i} \sum_{j>i} w_{i j} y_{i} y_{j}-\sum_{i} \lambda_{i} y_{i}
$$

This is a quadratic program in 0-1 variables with a $100 \%$ dense matrix of coefficients. Many algorithms and numerous heuristics have been proposed to solve it. In our experiments, we use a Variable Neighborhood Search (VNS) heuristic [49,50] as long as it can find a column with positive reduced cost. VNs is a metaheuristic, i.e., a framework for building heuristics, based on the idea of systematic change of neighborhood during the search. It explores progressively larger neighborhoods of the incumbent (or best known) solution in a probabilistic way. Therefore, often favorable characteristics of the incumbent will be kept and used to obtain promising neighboring solutions. VNS applies a local search routine repeatedly to get from these neighboring solutions to local optima.

When vNs fails to find an improving column, we use as exact method a simple branch and bound algorithm [51] or a recent algorithm using bounds based on semidefinite programming [52].

It is well known that column generation algorithms suffer from slow convergence particularly when the optimal solution is degenerate, i.e., when such a solution has many variables equal to 0, which is the case for clustering problems. Column generation algorithms also suffer from the plateau effect, i.e., the optimal solution keeps the same value for several or many iterations [53].

To alleviate these defects, one can use a variant of the stabilization methods for column generation due to du Merle et al. [54], which we call focussed column generation.

The principle is to identify, from a heuristic solution, a small region in the space of dual variables hopefully containing the optimal solution $\left(\lambda_{1}^{*}, \lambda_{2}^{*}, \ldots, \lambda_{n}^{*},\right)$. Then departures from this zone are penalized. 
To that effect, we first seek a good heuristic solution of the modularity maximization problem. Recently, Noack and Rotta [55] compared experimentally codes for eight heuristics. We used the SS+ML heuristic which is the best according to their experiments, i.e., Single-Step Greedy coarsening by Significance with MultiLevel Fast Greedy refinement (SS+ML). This heuristic solution can be further improved by a local application of modularity maximization to each pair of modules at a time. Then, a dual solution is derived from this last heuristic primal solution, and intervals hopefully containing the optimal values are determined. This is done by computing the increase (resp. the decrease) of the objective function value if a vertex is duplicated (resp. removed). Penalties for getting out of these intervals are imposed and progressively diminished until they get down to 0. Details on this method are given in [54].

\section{Modularity maximization by mixed 0-1 quadratic programming}

\subsection{Direct formulation}

Maximizing modularity by the clique partitioning approach discussed in Section 2 has a drawback: it replaces a usually sparse matrix of coefficients by a $100 \%$ dense one. An alternative approach is to work directly with a graph $G=(V, E)$ instead of the complete graph $K_{n}$. This was done by Xu, Tsoka and Papageorgiou [41] and leads to a $0-1$ mixed integer quadratic problem whose continuous relaxation is convex, and which can therefore be solved by CPLEX. We next recall the main elements of Xu et al.'s model as they provide the necessary background for a new column generation algorithm described in the second part of this section. Considering again the definition of $Q$ as a sum over modules of their modularities rewrite $Q$ as

$$
Q=\sum_{s}\left[a_{s}-e_{s}\right]=\sum_{s}\left[\frac{m_{s}}{m}-\left(\frac{D_{s}}{2 m}\right)^{2}\right]
$$

where $m_{s}$ denotes the number of edges in module $s$, i.e., the subgraph induced by $V_{s}$ and $D_{s}$ denotes the sum of degrees $k_{i}$ of the vertices of module $s$. Binary variables are then used to identify the modules to which each vertex and each edge belongs. To this effect, list all edges with a single index $r=1,2, \ldots, m$. Then, introduce the following variables,

$$
X_{r s}= \begin{cases}1 & \text { if edge } r \text { belongs to module } s \\ 0 & \text { otherwise }\end{cases}
$$

for $r=1,2, \ldots m$ and $s=1,2, \ldots M$ and

$$
Y_{i s}= \begin{cases}1 & \text { if vertex } i \text { belongs to module } s \\ 0 & \text { otherwise }\end{cases}
$$

The number of edges and sum of vertex degrees can then be expressed as:

$$
m_{s}=\sum_{r} X_{r s}
$$

and

$$
D_{s}=\sum_{i} k_{i} Y_{i s}
$$

A second series of constraints express that each vertex belongs to exactly one module:

$$
\sum_{s} Y_{i s}=1 \forall i=1,2, \ldots n
$$

A third series of constraints express that any edge $r=\left\{v_{i}, v_{j}\right\}$ with end vertices indiced by $i$ and $j$ can only belong to module $s$ if both of those end vertices belong to that module. 


$$
\begin{aligned}
& X_{r s} \leq Y_{i s} \quad \forall r=\left\{v_{i}, v_{j}\right\} \in E \\
& X_{r s} \leq Y_{j s} \quad \forall r=\left\{v_{i}, v_{j}\right\} \in E
\end{aligned}
$$

Note that these constraints are part of Fortet's [56] linearization of quadratic 0-1 programs. They impose that $X_{r s}=0$ if either $Y_{i s}$ or $Y_{j s}$ or both are equal to 0 . They do not impose that $X_{i s}$ is equal to 1 is $Y_{i s}=Y_{j s}=1$. Although the constraints $X_{r s} \geq Y_{i s}+Y_{j s}-1$ could be added to impose that, this is not necessary as adding an edge between vertices $i$ and $j$ when they are in the same module increases $m_{s}$ and hence $Q$.

The number of modules is a priori unknown; indicator variables $u_{s}=1$ if module $s$ is nonempty and $u_{s}=0$ otherwise are used. Then constraints

$$
u_{s} \leq u_{s-1} \quad \forall s \in 2,3, \ldots S
$$

where $S$ is an upper bound on the number of modules, are added and express that module $s$ can be nonempty only if module $s-1$ is so.

Consequently,

$$
\sum_{r} X_{r s} \geq u_{s}
$$

and

$$
\sum_{r} X_{r s} \leq(n-s+1) u_{s}
$$

The value $n-s+1$ in the latter constraints is due to the fact that each of the modules $1,2, \ldots s-1$ must be nonempty. In fact, Xu et al. use more general parametric formulae which allow imposing lower and upper bounds on the cardinality of the modules.

It is well-known that for any given solution to a clustering problem, alternative equivalent solutions can be obtained by simply re-indexing clusters. For an optimal solution with $M$ modules, $M$ ! equivalent solutions exist. Symmetry-breaking constraints for clustering problems can be found in the literature [57-59], and were used in [41]. Such symmetry breaking constraints notwithstanding, this problem has $M(n+m)$ binary variables and $M$ continuous variables, subject to $M(1+2 m+2 n)$ linear constraints.

\subsection{Column generation reformulation}

Once again, a linear programming master problem will be solved by the simplex algorithm where the entering column will be determined by solving an auxiliary problem. The master problem will be the same as in the previous column generation algorithm, i.e., its equations are given in (6)-(7),(9) of Section 2.2. The auxiliary problem will be different from the quadratic 0-1 program used in the clique partitioning formulation. It will be close to the formulation of $\mathrm{Xu}$ et al. [41] described in Section 3.1, but much simpler. As a single community is to be determined at a time, it can be written as follows:

$$
\begin{array}{cll}
\max _{x \in \mathbb{B}^{n}, D \in \mathbb{R}} & \sum_{r} \frac{x_{r}}{m}-\left(\frac{D}{2 m}\right)^{2}-\sum_{i} \lambda_{i} y_{i} & \\
\text { s.t. } & D=\sum_{i} k_{i} y_{i} & \\
& x_{r} \leq y_{i} & \forall r=\{i, j\} \in E \\
& x_{r} \leq y_{j} & \forall r=\{i, j\} \in E .
\end{array}
$$

As before, edges are indexed by $r$ and vertices by $i$ (or $j$ ). Variable $x_{r}$ is equal to 1 if edge $r$ belongs to the community which maximizes the objective function and to 0 otherwise. Similarly, $y_{i}$ is equal to 1 if the $i^{t h}$ vertex belongs to the community and 0 otherwise. The objective function is equal to the modularity of the community to be determined minus the scalar product of the current value $\lambda_{i}$ of the dual variables times 
the indicator variable $y_{i}$. Observe that this last term is the same as in the objective function of the auxiliary problem for clique partitioning. This is a mixed integer quadratic problem with $n+m$ binary variables and 1 continuous variable, in the objective function, subject to $2 m+1$ linear constraints. In the objective function there is a single concave nonlinear term. Clearly, the size of this auxiliary problem is much smaller than that of the direct formulation described in Section 3.1, particularly for large number of communities $M$. This auxiliary problem is first solved with a vNs heuristic as long as a column with a positive reduced cost can be found. When this is no more the case, CPLEX is called to find such a column or prove that there are no more.

\section{Computational comparison}

To compare the four algorithms described in the previous sections, we selected 11 test problems from the modularity maximization literature. Their names, orders and sizes are given in Table 1.

Table 1: Order and size of 11 test problems

\begin{tabular}{llcc}
\hline \hline Problem ID & Name & $\mathrm{n}$ & $\mathrm{m}$ \\
\hline \hline 1 & Zachary's karate club & 34 & 78 \\
2 & Dolphins social network & 62 & 159 \\
3 & Les Misérables & 77 & 254 \\
4 & A00_main & 83 & 135 \\
5 & protein p53 & 104 & 226 \\
6 & Books about US politics & 105 & 441 \\
7 & American College Football & 115 & 613 \\
8 & A01_main & 249 & 635 \\
9 & USAir97 & 332 & 2126 \\
10 & netscience_main & 379 & 914 \\
11 & Electronic Circuit (s838) & 512 & 819 \\
\hline \hline
\end{tabular}

The data of these test problems can be found in various databases mentioned in a recent paper from Noack and Rotta. Note that we always assume unit edge weights.

Results are given in Table 2. The first column gives the problem ID as in Table 1, the next six columns give the modularity value $(Q)$ and the number of modules $(M)$ for: (a) the solution obtained by NoackRotta's heuristic (NR Sol); (b) the improved solution obtained from Noack-Rotta's solution (Imp Sol); (c) the optimal solution (Opt Sol).

The remaining columns summarize the performance of the different exact algorithms:

- $C P R G$ : clique partitioning row generation of [39];

- $C P C G-H J M$ : clique partitioning column generation with the exact method of [51] for the auxiliary problem;

- $C P C G$-BE: clique partitioning column generation with the exact method of [52] for the auxiliary problem; 0-1 mixed integer programming formulation of [41];

- 0-1 MICG: 0-1 mixed integer column generation.

For CPRG column, the CPU time is reported when possible or an "OM" is used to indicate that the program reaches the memory limit. The formulation of [39] is implemented in AMPL [60] and solved using the "lazy constraints" feature of CPLEX. We do not present the results for the formulation of [16] as it is much more time consuming. For example, the solution of Problem 1 takes 674 seconds instead of 0.23 second.

For the 0-1 MIQP column, we give the CPU time reported in [41] or indicate by "NA" the problem not considered in [41]. It was not possible for us to obtain comparable computing time as the parameters setting used to obtain these results were not detailed in the paper.

For the column generation algorithms, the table contains CPU time as well as, in parentheses, the number of calls for the exact algorithm for the auxiliary problem followed by the number of calls to the heuristic. The term "OT" means that the optimal solution could not be found after more than 100000 seconds. 
Table 2: Results of the comparison between algorithms for modularity maximization. Best results are reported in boldface.

\begin{tabular}{|c|c|c|c|c|c|c|c|c|c|c|c|c|}
\hline \multirow{2}{*}{$\begin{array}{l}\mathrm{Pb} . \\
\mathrm{ID}\end{array}$} & \multicolumn{2}{|c|}{ NR Sol } & \multicolumn{2}{|c|}{ Imp Sol } & \multicolumn{2}{|c|}{ Opt Sol } & \multirow[t]{2}{*}{$C P R G$} & \multicolumn{2}{|c|}{$C P C G-$} & \multirow[t]{2}{*}{$0-1 M I Q P$} & \multicolumn{2}{|c|}{$0-1 M I C G$} \\
\hline & $Q$ & $M$ & $Q$ & $M$ & $Q$ & $M$ & & $H J M$ & $B E$ & & & \\
\hline$\overline{11}$ & 0.4198 & $\overline{4}$ & $\overline{0.4198}$ & $\overline{4}$ & 0.4198 & $\overline{4}$ & 0.23 & $(3 / 7)$ & $(3 / 7)$ & 1.03 & $\overline{0.34}$ & $(3 / 8)$ \\
\hline 2 & 0.5238 & 4 & 0.5285 & 5 & 0.5285 & 5 & 9.70 & $(1 / 13)$ & $(1 / 13)$ & 197.89 & 7.75 & $(1 / 13)$ \\
\hline 3 & 0.5600 & 6 & 0.5600 & 6 & 0.5600 & 6 & 5.07 & $(1 / 13)$ & $(1 / 13)$ & 55.58 & 7.26 & $(1 / 13)$ \\
\hline 4 & 0.56749 & 9 & 0.5283 & 9 & 0.5309 & 9 & 17.09 & $(1 / 26)$ & 262.13 & NA & 3.66 & $(1 / 26)$ \\
\hline 5 & 0.5322 & 7 & 0.5350 & 6 & 0.5351 & 7 & 4164.15 & $(1 / 44)$ & 351.87 & 1844.31 & 11.60 & $(1 / 44)$ \\
\hline 6 & 0.5269 & 4 & 0.5272 & 5 & 0.5272 & 5 & 663.05 & 318.99 & 32287.23 & $\mathrm{NA}$ & 45.65 & $(2 / 13)$ \\
\hline 7 & 0.6002 & 10 & 0.6046 & 10 & 0.6046 & 10 & 282.58 & $\mathrm{OT}$ & $593.01 \quad(1 / 8)$ & NA & 249.41 & $(1 / 8)$ \\
\hline 8 & 0.6203 & 12 & 0.6203 & 12 & 0.6329 & 14 & $\mathrm{OM}$ & OT & $\mathrm{OT}$ & NA & 1014.48 & $(12 / 145)$ \\
\hline 9 & 0.3658 & 6 & 0.3660 & 6 & 0.3682 & 6 & OM & OT & OT & NA & 16216.77 & $(3 / 104)$ \\
\hline 10 & 0.8474 & 19 & 0.8485 & 19 & 0.8486 & 19 & $\mathrm{OM}$ & $\mathrm{OT}$ & OT & NA & 1615.14 & $(12 / 66)$ \\
\hline 11 & 0.8162 & 16 & 0.8166 & 16 & 0.8194 & 12 & $\mathrm{OM}$ & OT & OT & NA & 7655.56 & $(140 / 225)$ \\
\hline
\end{tabular}

All results are in seconds of CPU. Except for 0-1 MIQP, all results were obtained on a dual processor computer Intel Pentium computer with $3.20 \mathrm{GHz}, 2 \mathrm{Mb}$ cache memory, $3 \mathrm{~GB}$ RAM running under Linux. ILOG CPLEX 10.110 was used for the linear programming part of all algorithms.

From Table 2, it appears that:

- surprisingly, in view of the many heuristics proposed for modularity maximization, those applied here seldom reach the optimal value: in 2 cases out of 11 of Noack and Rotta and 5 cases out of 11 for the improved heuristic;

- both row-generation and column-generation algorithm based on reformulation of modularity maximization as a clique partitioning problem are competitive for small instances, but become too time or memory consuming for larger ones due to the rapid increase in the number of variables and constraints;

- for the largest instances, the $0-1 M I C G$ algorithm appears to be the only one able to find an optimal solution;

- the reduction in resolution times in comparison of the results of [41] are very substantial: the use of 0-1 MICG algorithm divides these times by a factor of 3 to 159 ;

- the size of the largest problem solved is raised from 105 entities to 512 entities.

In summary, the column generation algorithm reformulated from [41] direct formulation (0-1 MICG) appears to be the best choice since its computing time is comparable for small instances, lower for medium instances and is the only algorithm able to solve large instances to optimality.

\section{Conclusions}

In this paper, we have studied exact maximization of the modularity of a network according to the definition of Newman and Girvan. Two approaches were proposed previous to this work: on the one hand the row generation algorithm of [39] to which modularity maximization can be reduced and on the other hand the direct formulation of $\mathrm{Xu}$ et al. [41]. We have proposed column generation algorithms based on these two approaches and performed a computation comparison on a series of well-known problems from the literature. These results show that: (i) while row generation is fast for small problems, it is outperformed by column generation for larger ones; (ii) the column generation algorithm based on direct formulation performs best for the larger problems; (iii) size of the larger problems has substantially augmented: the largest problem solved to date has 512 entities versus 105 entities before; (iv) computation times are substantially reduced. While it would of course be desirable to solve exactly larger problems, the results obtained by the proposed algorithm already appear to be useful for the several reasons detailed in the introduction.

It is easy to see that the first two approaches can also be applied to several variants of the standard modularity definition, i.e., weighted networks, directed networks, and networks which are both directed and weighted [61]. Moreover, the column generation framework is easily adaptable to yield exact algorithms 
for other definitions of communities than those of Newman and Girvan, allowing comparison which do not depend on the specific heuristic used.

Among topics for future research are (i) the design of better heuristics, (ii) the use of cutting planes in the solution of the master problem and/or the auxiliary problem, (iii) a further study of the properties of the auxiliary problem which might lead to improvements in its resolution. The importance of this last point stems from the fact that the proportion of the resolution time devoted to the resolution of the auxiliary problem tends to increase with problem size.

\section{References}

[1] P. Hansen and B. Jaumard, "Cluster analysis and mathematical programming," Mathematical Programming, vol. 79, pp. 191-215, 1997.

[2] A. Jain, M. Murty, and P. Flynn, "Data clustering: A review," ACM Computing Surveys, vol. 31, no. 3, pp. 264$323,1999$.

[3] L. Kaufman and P. Rousseeuw, Finding Groups in Data. An Introduction to Cluster Analysis. Wiley Series in Probability and Statistics, 2005.

[4] B. Mirkin, Clustering for Data Mining: A Data Recovery Approach. Boca Raton, FL: Chapman and Hall/CRC, 2005.

[5] F. Radicchi, C. Castellano, F. Cecconi, V. Loreto, and D. Parisi, "Defining and identifying communities in networks," Proceedings of the National Academy of Sciences, USA, vol. 101, no. 9, pp. 2658-2663, 2004.

[6] Z. Li, S. Zhang, R.-S. Wang, X.-S. Zhang, and L. Chen, "Quantitative function for community detection," Physical Review E, vol. 77, p. 036109, Mar 2008.

[7] A. D. Medus and C. O. Dorso, "Alternative approach to community detection in networks," Physical Review E, vol. 79, p. 066111, Jun 2009.

[8] S. Cafieri, P. Hansen, and L. Liberti, "Edge ratio and community structure in networks," Physical Review E, vol. 81, p. 026105, Feb 2010.

[9] M. Newman and M. Girvan, "Finding and evaluating community structure in networks," Physical Review E, vol. 69, no. $026133,2004$.

[10] C. Massen and J. Doye, "Identifying communities within energy landscapes," Physical Review E, vol. 71, no. 046101, 2005.

[11] S. Fortunato and M. Barthelemy, "Resolution limit in community detection," Proceedings of the National Academy of Sciences, USA, vol. 104, no. 1, pp. 36-41, 2007.

[12] X. S. Zhang, R. S. Wang, Y. Wang, J. Wang, Y. Qiu, L. Wang, and L. Chen, "Modularity optimization in community detection of complex networks," Europhysics Letters, vol. 87, no. 3, p. 38002, 2009.

[13] S. Cafieri, P. Hansen, and L. Liberti, "Loops and multiple edges in modularity maximization of networks," Physical Review E, vol. 81, p. 046102, 2010.

[14] J. Kumpula, J. Saramaki, K. Kaski, and J. Kertesz, "Limited resolution and multiresolution methods in complex network community detection," Fluctuation and Noise Letters, vol. 7, no. 3, pp. L209-L214, 2007.

[15] X.-S. Zhang and R.-S. Wang, "A Constrained Optimization Method for Community Detection," in Proceedings of the Third International Symposium on Optimization and Systems Biology, Zhangjiajie, China, September 20-22, 2009, pp. 142-150, 2009.

[16] U. Brandes, D. Delling, M. Gaertler, R. Görke, M. Hoefer, Z. Nikoloski, and D. Wagner, "On modularity clustering," IEEE Transactions on Knowledge and Data Engineering, vol. 20, no. 2, pp. 172-188, 2008.

[17] M. Newman, "Fast algorithm for detecting community structure in networks," Physical Review E, vol. 69, no. 066133, 2004.

[18] A. Clauset, M. Newman, and C. Moore, "Finding community structure in very large networks," Physical Review $E$, vol. 70, 2004.

[19] L. Danon, A. Diaz-Guilera, and A. Arenas, "The effect of size heterogeneity on community identification in complex networks," Journal of Statistical Mechanics, vol. P11010, 2006.

[20] K. Wakita and T. Tsurumi, "Finding community structure in mega-scale social networks," Tech. Rep. 0702048v1, arXiv, 2007.

[21] V. Blondel, J.-L. Guillaume, R. Lambiotte, and E. Lefebvre, "Fast unfolding of communities in large networks," Journal of Statistical Mechanics, p. P10008, 2008. 
[22] R. Guimerà and A. Amaral, "Functional cartography of complex metabolic networks," Nature, vol. 433, pp. 895900, 2005.

[23] A. Medus, G. Acuna, and C. Dorso, "Detection of community structures in networks via global optimization," Physica A, vol. 358, pp. 593-604, 2005.

[24] S. Lehmann and L. Hansen, "Deterministic modularity optimization," European Physical Journal B, vol. 60, pp. 83-88, 2007.

[25] M. Tasgin, A. Herdagdelen, and H. Bingol, "Community detection in complex networks using genetic algorithms," arXiv:0711.0491, 2007.

[26] J. Duch and A. Arenas, "Community identification using extremal optimization," Physical Review E, vol. 72 -027104 , no. 2, 2005.

[27] M. Newman, "Modularity and community structure in networks," Proceedings of the National Academy of Sciences, USA, pp. 8577-8582, 2006.

[28] T. Richardson, P. Mucha, and M. Porter, "Spectral tripartitioning of networks," Physical Review E, vol. 80, p. 036111, Sep 2009.

[29] Y. Sun, B. Danila, K. Josic, and K. E. Bassler, "Improved community structure detection using a modified fine-tuning strategy," Europhysics Letters, vol. 86, no. 28004, 2009.

[30] G. Agarwal and D. Kempe, "Modularity-maximizing graph communities via mathematical programming," The European Physical Journal B, vol. 66, no. 3, pp. 409-418, 2008.

[31] S. Boccaletti, M. Ivanchenko, V. Latora, A. Pluchino, and A. Rapisarda, "Detecting complex network modularity by dynamical clustering," Physical Review E, vol. 75, no. 045102(R), 2007.

[32] H. Djidjev, "A scalable multilevel algorithm for graph clustering and community structure detection," Lecture Notes in Computer Science, vol. 4936, 2008.

[33] J. Mei, S. He, G. Shi, Z. Wang, and W. Li, "Revealing network communities through modularity maximization by a contraction-dilation method," New Journal of Physics, vol. 11, no. 043025, 2009.

[34] P. Schuetz and A. Caflisch, "Efficient modularity optimization by multistep greedy algorithm and vertex mover refinement," Physical Review E, vol. 77, no. 046112, 2008.

[35] Y. Niu, B. Hu, W. Zhang, and M. Wang, "Detecting the community structure in complex networks based on quantum mechanics," Physica A, vol. 387, no. 24, pp. 6215-6224, 2008.

[36] D. Chen, Y. Fu, and M. Shang, "A fast and efficient heuristic algorithm for detecting community structures in complex networks," Physica A, vol. 388, no. 13, pp. 2741-2749, 2009.

[37] J. Ruan and W. Zhang, "Identifying network communities with a high resolution," Physical Review E, vol. 77, no. 016104, 2008.

[38] Y. Fan, M. Li, P. Zhang, J. Wu, and Z. Di, "Accuracy and precision of methods for community identification in weighted networks," Physica A, vol. 377, no. 1, pp. 363-372, 2007.

[39] M. Grötschel and Y. Wakabayashi, "A cutting plane algorithm for a clustering problem," Mathematical Programming, vol. 45, pp. 59-96, 1989.

[40] M. Grötschel and Y. Wakabayashi, "Facets of the clique partitioning polytope," Mathematical Programming, vol. 47, pp. 367-387, 1990.

[41] G. Xu, S. Tsoka, and L. Papageorgiou, "Finding community structures in complex networks using mixed integer optimization," Eur. Physical Journal B, vol. 60, pp. 231-239, 2007.

[42] S. Cafieri, P. Hansen, and L. Liberti, "Improving heuristics for network modularity maximization using an exact algorithm," in MatHeuristics 2010, (Heidelberg), Springer, accepted.

[43] ILOG, ILOG CPLEX 11.0 User's Manual. ILOG S.A., Gentilly, France, 2008.

[44] G. Dantzig, R. Fulkerson, and S. Johnson, "Solution of a large-scale traveling salesman problem," Operations Research, vol. 2, pp. 393-410, 1954.

[45] D. Applegate, R. Bixby, V. Chvtal, W. Cook, D. Espinoza, M. Goycoolea, and K. Helsgaun, "Certification of an optimal tsp tour through 85,900 cities," Operations Research Letters, vol. 37, no. 1, pp. 11-15, 2009.

[46] T. Achterberg, T. Koch, and A. Martin, "Branching rules revisited," Operations Research Letters, vol. 33, no. 1, pp. $42-54,2005$.

[47] O. Du Merle, P. Hansen, B. Jaumard, and N. Mladenovic, "An interior point algorithm for minimum sum-ofsquares clustering," Siam Journal on Scientific Computing, vol. 21, pp. 1485-1505, Apr 272000.

[48] D. Aloise, P. Hansen, and L. Liberti, "An Improved Column Generation Algorithm for Minimum Sum-of-squares Clustering ," Mathematical Programming, to appear.

[49] N. Mladenovic and P. Hansen, "Variable neighborhood search," Computers and Operations Research, vol. 24, no. 11, pp. 1097-1100, 1997. 
[50] P. Hansen and N. Mladenovic, "Variable neighborhood search: Principles and applications," European Journal of Operational Research, vol. 130, no. 3, pp. 449-467, 2001.

[51] P. Hansen, B. Jaumard, and C. Meyer, "A simple enumerative algorithm for unconstrained 0-1 quadratic programming," Les Cahiers du GERAD, G-2000-59, 2000.

[52] A. Billionnet and S. Elloumi, "Using a mixed integer quadratic programming solver for the unconstrained quadratic 0-1 problem," Mathematical Programming, vol. 109, pp. 55-68, JAN 2007.

[53] F. Vanderbeck and M. Savelsbergh, "A generic view of dantzig-wolfe decomposition in mixed integer programming," Operations Research Letters, vol. 34, no. 3, pp. 296-306, 2006.

[54] O. du Merle, D. Villeneuve, J. Desrosiers, and P. Hansen, "Stabilized column generation," Discrete Mathematics, vol. 194, pp. 229-237, 1999.

[55] A. Noack and R. Rotta, "Multi-level algorithms for modularity clustering," Lecture notes in computer sciences, vol. 5526, pp. 257-268, 2009.

[56] R. Fortet, "L'algèbre de Boole et ses applications en Recherche Opérationnelle," Cahier du centre d'études en recherche opérationnelle, vol. 1, no. 4, pp. 5-36, 1959.

[57] G. Klein and J. Aronson, "Optimal clustering: A model and method," Naval Research Logistics, vol. 38, no. 3, pp. 447-461, 2006.

[58] H. Sherali and J. Desai, "A global optimization rlt-based approach for solving the hard clustering problem," Journal of Global Optimization, vol. 32, pp. 281-306, 2005.

[59] F. Plastria, "Formulating logical implications in combinatorial optimisation," European Journal of Operational Research, vol. 140, no. 2, pp. 338-353, 2002.

[60] R. Fourer and D. Gay, The AMPL Book. Pacific Grove: Duxbury Press, 2002.

[61] S. Fortunato, "Community detection in graphs," Physics reports, vol. 486, no. 3-5, pp. 75-174, 2010. 